\title{
On the Tag Detection in Near-field UHF RFID Applications
}

\author{
Andrea Michel*(1), Marcos R. Pino ${ }^{(2)}$, Paolo Nepa ${ }^{(1)}$, and Giuliano Manara ${ }^{(1)}$ \\ (1) Dept. Information Engineering, University of Pisa, 56122 Pisa, Italy \\ (2) Dept. of Electrical Engineering, University of Oviedo, E-33203 Gijón, Spain
}

\begin{abstract}
The UHF RFID tag detection is here investigated when the transponder and the reader antenna are placed close each other. Differently from long-range RFID applications, in near-field applications the field distribution generated by the reader antenna as well as the tag layout play an important role in the tag detection. In this paper, considerations on the tag readability are proposed on the basis of both simulated and measured results.
\end{abstract}

\section{Introduction}

It is known that Ultra-High Frequency (UHF) Radio Frequency Identification (RFID) systems allow for a high read- and data-rate and for small size tags. Moreover, NearField (NF) UHF RFID systems have been introduced to maximize and confine the electric and magnetic fields within a limited volume around the reader. The far-field radiation efficiency is required to be quite low, so reducing the detection of tags placed far from the reader (false positive issues).

In the scientific literature, a large number of antennas for NF UHF RFID readers have been proposed [1-7]. Typically, the antenna design is based on both impedance matching and radiative characteristics such as gain and polarization. However, for near-field applications, also the electric and magnetic field distributions must be investigated to make the tag detection as independent as possible on the tag position and orientation with respect to the reader antenna. In several papers, the electric field distributions are analyzed, which are reasonably related to the dipole-like tag detection. On the other hand, the magnetic field distribution may be a key parameter for the loop-like tags detection. However, for hybrid tag layout, both the electric and magnetic fields have to be taken into account. Differently from the fields distributions, in some papers the Power Transfer Efficiency has been taken into account to qualitatively and quantitative predict the tag detection [8-9].

In this paper, a simulated and measured analysis is presented to find a relation between simulated and measured antenna characteristics. Doing so, the systemlevel performance in terms of tag detection can be improved during the antenna design process, especially when the tag typology is known.

\section{Layout}

The presented analysis has been carried out by considering the Reconfigurable Modular Antenna recently proposed for NF UHF RFID smart point readers [7]. In this layout (Figure 1), a spiral microstrip line ends on an absorptive RF switch. When the spiral line ends on a matched load, strong and uniform electric and magnetic fields are generated up to a few centimeters from the antenna surface (near-field reactive region). On the basis of the specific operative scenario, the spiral microstrip line can feed an array of four curved slot antennas which share the same aperture (aperture-shared antenna configuration). Thus, the read range is extended up to a few tens of centimeters from the antenna surface (radiative near-field region), yet radiating a relatively low field in the antenna far-field region as required by antennas for desktop readers.

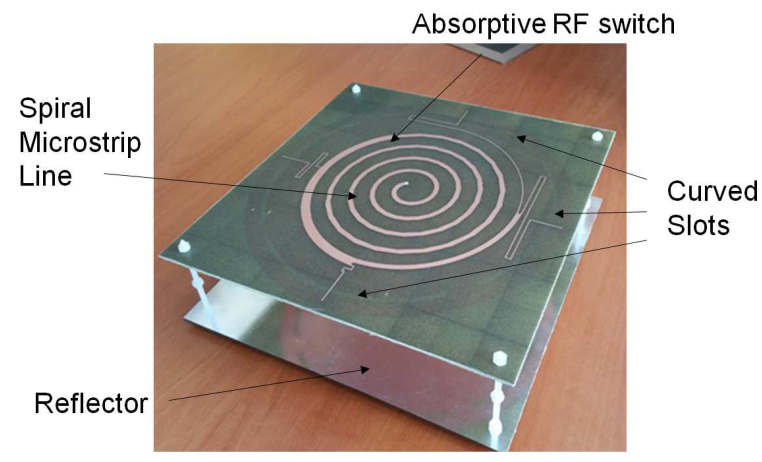

Figure 1. Fabricated prototype of the Reconfigurable Modular Antenna proposed in [7].

As previously discussed, a tag detection prediction through numerical simulations would be useful to optimize the radiating element layout, especially when the RFID tag typology is a priori known. For this reason, four distributions have been computed or measured, aiming to find a relation among simulated and measured antenna performance. Specifically, the following distributions have been compared by setting a distance of $10 \mathrm{~cm}$ from the antenna surface and an operating frequency of $865 \mathrm{MHz}$ :

- Simulated field distribution

- Measured field distribution

- Simulated Power Transfer Efficiency (PTE) distribution

- Measured Received Signal Strength Indicator (RSSI) distribution 
The antenna simulated performance has been obtained by using the commercial numerical tool CST Microwave Studio $^{\circledR}$. The simulated field distribution can be compared with the measured field distribution measured in the nearfield chamber at the University of Oviedo.

The simulated PTE between the reader and the tag antennas has been also computed to qualitatively estimate the power delivered to the tag chip when varying the tag position and orientation. In Figure 2, the reader-tag configuration considered to compute the PTE is shown, when a dipolelike (Figure 2a) or a loop-like (Figure 2b) tag layouts are included in the simulated scenarios.

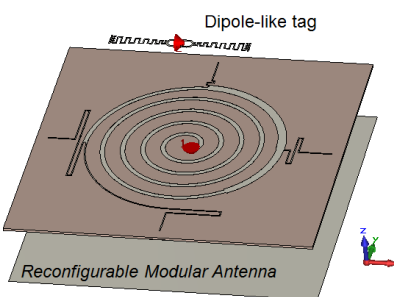

(a)

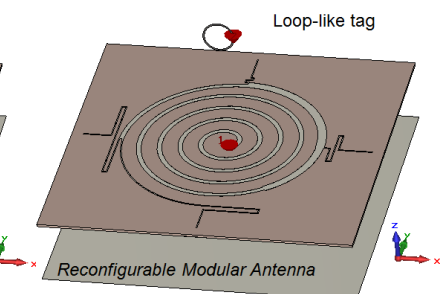

(b)
Figure 2. Simulated model used to compute the PTE between the Reconfigurable Modular Antenna presented in [7] and a (a) dipole-like and (b) loop-like tag typology.

The PTE is defined as the ratio between the power dissipated in the load of the tag antenna (chip), $P_{T}$, and the input power accepted by the reader antenna, $P_{R}$. Specifically, by considering the tag, the reader antenna, and the space between them, as a two-port network, the $Z_{i j}$ entries of the impedance matrix $\underline{\underline{Z}}$ can be computed, as described in [8]. Then, the PTE can be calculated as (1), where $Z_{I N}$ is the input impedance of the two-ports network when the latter is connected to $Z_{L}$.

$$
P T E=\frac{P_{T}}{P_{R}}=\frac{1 / 2 \Re\left\{Z_{L}\right\}\left|I_{2}\right|^{2}}{1 / 2 \Re\left\{Z_{I N}\right\}\left|I_{1}\right|^{2}}=\left|Z_{21}\right|^{2} \frac{\Re\left\{Z_{L}\right\} / \Re\left\{Z_{I N}\right\}}{\left|Z_{L}+Z_{22}\right|^{2}}
$$

Furthermore, for a given tag and reader antenna layout, the PTE can be measured by following the procedure described in [9]. However, in this paper, the simulated PTE is compared with the measured RSSI distributions obtained by using a commercial reader (CAEN RFID Ion, Model $\mathrm{R} 4301 \mathrm{P})$. The reader output power has been set to $20 \mathrm{dBm}$, and the RSSI values have been measured by varying the tag position with respect to the reader antenna, at a distance of $10 \mathrm{~cm}$.

\section{Results and conclusions}

In Figure 3, the four normalized distributions have been compared. Specifically, the dipole-like Inlay UH100 LABID tag has been considered, so the analysis has focused on the electric field distribution. In Figure 3a-b, the simulated and measured normalized electric field distributions are plotted. The comparison between these distributions provides a qualitative indication on the reliability of the simulated model and the measurement setup. Moreover, in Figure $3 \mathrm{c}$ and Figure $3 \mathrm{~d}$ the simulated PTE and the measured RSSI normalized distributions are shown, respectively. For this example, it is worth noting that the tag detection can be qualitatively predicted by investigating the PTE through numerical analysis. Moreover, since a dipole-like tag has been considered, also the simulated electric field can be analyzed during the reader antenna design process to improve the system-level performance of the entire RFID system. The analysis can be repeated by considering loop-like tags, aiming to derive similar conclusions.

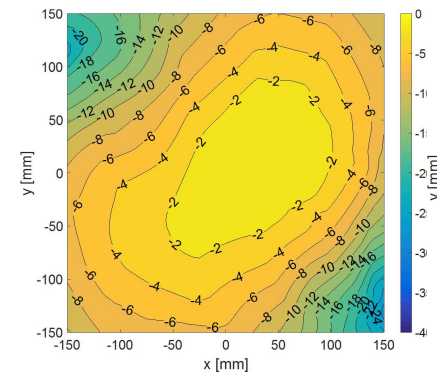

(a)

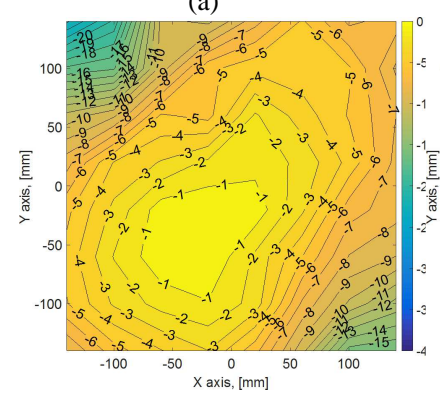

(c)

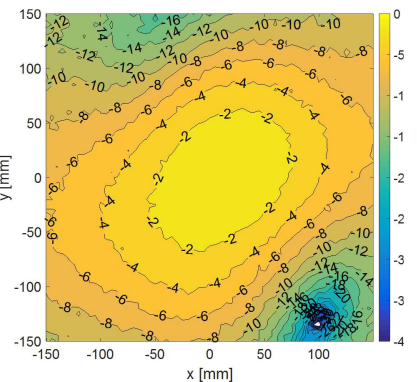

(b)

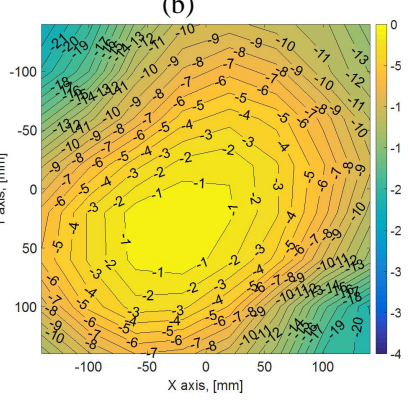

(d)
Figure 3. Normalized (a) simulated and (b) measured electric field at $865 \mathrm{MHz}$, at a distance of $10 \mathrm{~cm}$ from the antenna surface, when the Modular Antenna Configuration is enabled. Normalized (c) numerical PTE and (d) measured RSSI distribution at a distance of $10 \mathrm{~cm}$ from the antenna surface when the LAB-ID Inlay UH100 tag is considered.

\section{Acknowledgements}

This work was supported in part by the Spanish 'Ministerio de Economia y Competitividad' under the project MIRIIEM with ref. TEC-2015-540005-P and by the Gobierno del Principado de Asturias / FEDER under project GRUPIN14-114.

\section{References}

1. J. Shi, X. Qing, and Z. N. Chen, "Electrically Large Zero-Phase-Shift Line Grid-Array UHF Near-Field RFID Reader Antenna," IEEE Trans. Antennas Propag., vol. 62, no. 4, pp. 2201-2208, Apr. 2014. 
2. C. R. Medeiros, J. R. Costa, and C. A. Fernandes, "RFID Reader Antennas for Tag Detection in SelfConfined Volumes at UHF," IEEE Antennas Propag. Mag., vol. 53, no. 2, pp. 39-50, Apr. 2011.

3. A. Michel, A. Buffi, R. Caso, P. Nepa, G. Isola, and H. T. Chou, "Design and performance analysis of a planar antenna for near-field UHF-RFID desktop readers," in Microwave Conference Proceedings (APMC), 2012 AsiaPacific, 2012, pp. 1019-1021.

4. A. Michel, R. Caso, A. Buffi, P. Nepa, and G. Isola, "Meandered TWAS array for near-field UHF RFID applications," Electron. Lett., vol. 50, no. 1, pp. 17-18, Jan. 2014.

5. A.S. Andrenko, "Optimized near-field antenna for UHF RFID smart shelf applications," IEEE International Symposium on Antennas and Propagation \& USNC/URSI National Radio Science Meeting, pp.1576-1577, 19-24 July 2015

6. A. Michel, R. Caso, A. Buffi and P. Nepa, "Multifunctional modular antenna for near-field ultra-high frequency radio frequency identification readers," in IET Microwaves, Antennas \& Propagation, vol. 10, no. 8, pp. 843-849, 2016

7. A. Michel; M. Rodriguez-Pino; P. Nepa, "Reconfigurable Modular Antenna for Near-Field UHF RFID Smart Point Readers," IEEE Trans. Antennas and Propag., vol. 65, no. 2, pp. 1-9, Feb. 2017

8. A. Buffi, A. Michel, R. Caso and P. Nepa, "Near-field coupling in UHF-RFID systems," 2013 International Symposium on Electromagnetic Theory, Hiroshima, 2013, pp. 408-411.

9. A. C. de Souza, Y. Duroc, T. P. Vuong and A. Luce, "Quantitative evaluation of power transfer efficiency of UHF RFID passive systems," in Electronics Letters, vol. 51, no. 12, pp. 932-933, 6112015 